\title{
Evidenza RMN di metastasi ipofisaria isolata da carcinoma polmonare
}

\author{
Dario De Alcubierre ${ }^{1}$. Giulia Puliani ${ }^{1}$. Emilia Sbardella ${ }^{1}$. Riccardo Pofi ${ }^{1}$ - Alessia Cozzolino ${ }^{1}$. Marta Tenuta ${ }^{1}$. \\ Carlotta Pozza ${ }^{1}$. Francesca Gianco ${ }^{2}$. Felice Giangaspero ${ }^{2}$. Elisa Giannetta ${ }^{1}$. Andrea Lenzi ${ }^{1}$. Andrea M. Isidori ${ }^{1}$
}

Una signora di 80 anni era stata trattata chirurgicamente nel 2013 per un adenocarcinoma polmonare moderatamente differenziato di tipo misto, infiltrante lo stroma, adiacente al quale si rilevava la presenza di un focolaio distinto riferibile a tumorlet di $1,5 \mathrm{~mm}$ di diametro, positivo alla colorazione per CD56, negativo per Sinaptofisina, CD7, TTF1, di cui veniva confermata la natura neuroendocrina. Circa tre anni dopo, nel luglio 2016, per insorgenza di calo del visus, diplopia e vomito, effettuava una RMN encefalo, che evidenziava una neoformazione solida evolutiva della sella turcica di $22 \times 27$ $\times 44 \mathrm{~mm}$, che comprimeva e dislocava il chiasma ottico e il terzo ventricolo (Fig. 1a). Dati i sintomi visivi e l'idrocefalo, considerate le buone condizioni generali, veniva sottoposta a intervento chirurgico di asportazione trans-sfenoidale della lesione (Fig. 1b). Lo studio istologico e immunoistochimico evidenziava, in sede sellare intradurale, la presenza di tessuto tumorale di origine epiteliale, positivo alle colorazioni per CK AF1/AF3 e CK MNF116 (Fig. 2). Di tale lesione epiteliale, nonostante la negatività per TTF1 e Napsin A, veniva refertata la verosimile pertinenza metastatica polmonare, data la storia clinica della paziente.

Ai successivi controlli postoperatori la paziente lamentava cefalea, offuscamento del visus, mentre le RMN evidenziavano persistenza e successivo aumento dimensionale della lesione residua fino a $20 \times 26 \times 40 \mathrm{~mm}$, con compressione del chiasma ottico.

D. De Alcubierre

dario.dealcubierre@uniroma1.it

1 Dipartimento di Medicina Sperimentale, Università "Sapienza" di Roma, Roma, Italia

2 Dipartimento di Medicina Molecolare, Università "Sapienza" di Roma, Roma, Italia
Pertanto, la paziente veniva sottoposta a radioterapia stereotassica frazionata per una dose totale di $25 \mathrm{~Gy}$, con sostanziale stabilità del tessuto patologico ai controlli RMN, fino all'exitus avvenuto a gennaio 2018 .

Questo caso di metastasi ipofisaria isolata evidenzia come il trattamento multimodale sembra essere l'approccio preferibile per garantire la stabilità di lesioni dal comportamento aggressivo, attraverso una combinazione di debulking neurochirurgico e radioterapia $[1,2]$.

Funding Note Open Access funding provided by Università degli Studi di Roma La Sapienza within the CRUI-CARE Agreement.

Conflitto di interessi Gli autori Dario De Alcubierre, Giulia Puliani, Emilia Sbardella, Riccardo Pofi, Alessia Cozzolino, Marta Tenuta, Carlotta Pozza, Francesca Gianco, Felice Giangaspero, Elisa Giannetta, Andrea Lenzi e Andrea M. Isidori dichiarano di non avere conflitti di interesse.

Consenso informato La figlia della paziente, previo colloquio informativo, ha fornito il proprio consenso alla pubblicazione delle immagini.

Studi sugli animali Gli autori di questo articolo non hanno eseguito studi sugli animali.

Nota della casa editrice Springer Nature rimane neutrale in riguardo alle rivendicazioni giurisdizionali nelle mappe pubblicate e nelle affiliazioni istituzionali.

Open Access This article is licensed under a Creative Commons Attribution 4.0 International License, which permits use, sharing, adaptation, distribution and reproduction in any medium or format, as long as you give appropriate credit to the original author(s) and the source, provide a link to the Creative Commons licence, and indicate if changes were made. The images or other third party material in this article are included in the article's Creative Commons licence, unless indicated otherwise in a credit line to the material. If material is not included in the article's Creative Commons licence and your intended use is not permitted by statutory regulation or exceeds the permitted use, you will need to obtain permission directly from the copyright holder. To view a copy of this licence, visit http://creativecommons.org/licenses/by/4.0/. 

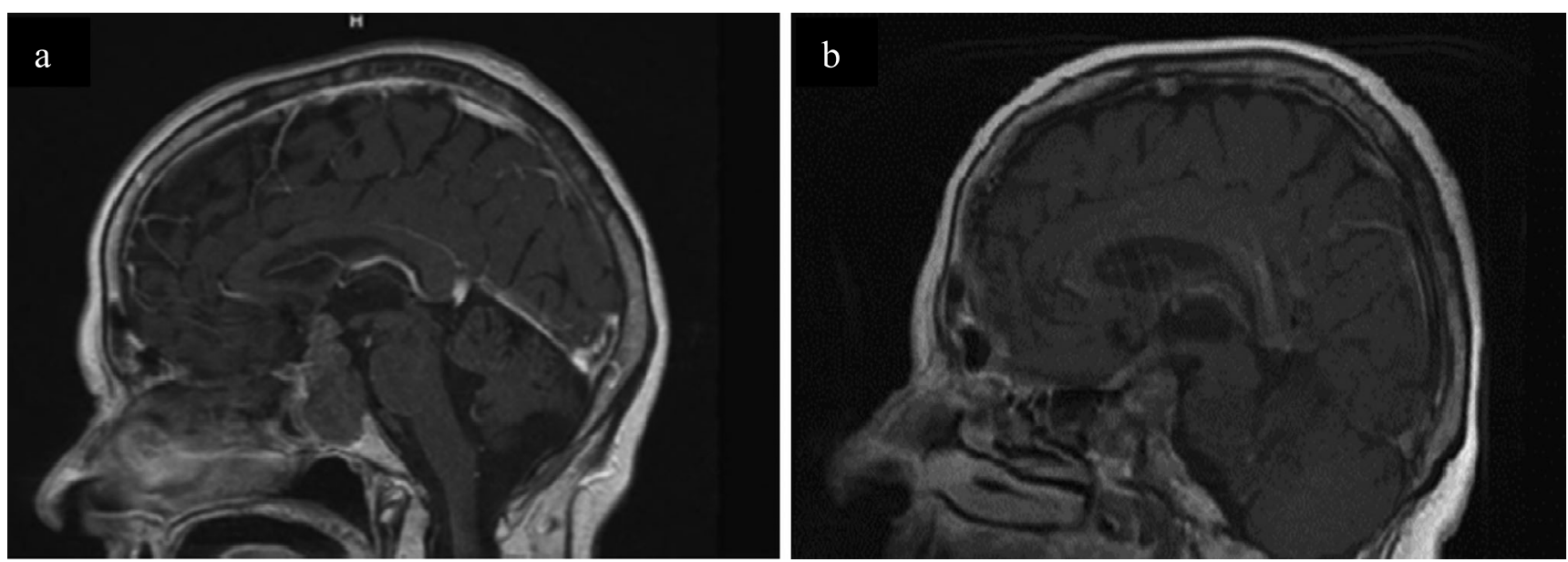

Fig. 1 Scansione sagittale RM T1-pesata, pre-neurochirurgia (a) e post-neurochirurgia (b)

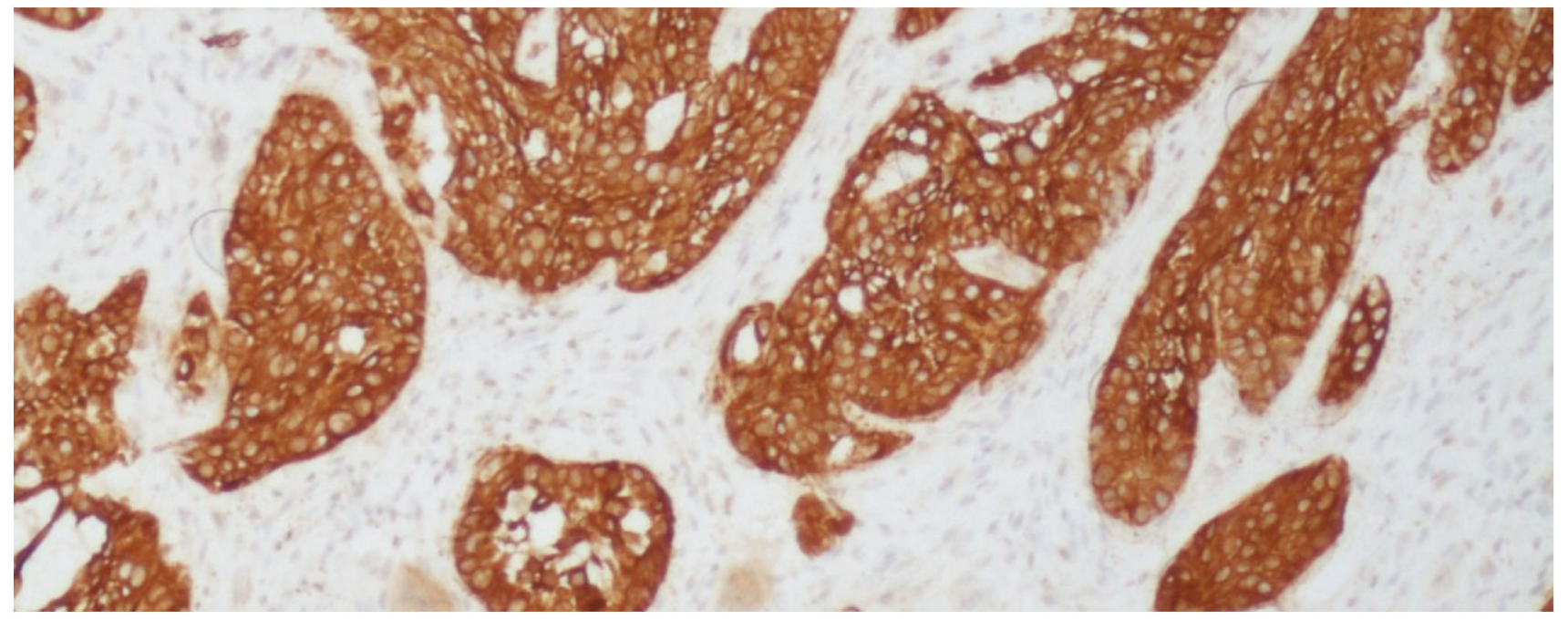

Fig. 2 Positività del tessuto patologico sellare alla colorazione per $\mathrm{CK}$

\section{Bibliografia}

1. Ng S, Fomekong F, Delabar V et al (2020) Current status and treatment modalities in metastases to the pituitary: a systematic review. J Neurooncol 146(2):219-227
2. Patel KR, Zheng J, Tabar V et al (2019) Extended survival after surgical resection for pituitary metastases: clinical features, management, and outcomes of metastatic disease to the sella. The Oncologist 25(5):e789-e797 